Attendance logs and feedback forms were available for 53 sessions. A total of 2369 attendances were logged, with a median of 41 attendees per session (IQR 31-54). Attendees from 22 countries have participated in these sessions.

A total of 810 survey feedback forms were received, with a median of 14 forms received per session (IQR 10-18). 32\% were filled in by PGHAN Grid trainees, $23 \%$ by consultants, $15 \%$ by clinical fellows. Allied Health Professionals (AHPs) comprised $6 \%$ of feedback returns. $54 \%$ of survey feedback respondents accessed the teaching sessions from home.

An average of 98\% (95\% CI 96.3-99.2) survey respondents strongly agreed/agreed that the sessions were relevant to their learning. $97 \% \quad(95 \%$ CI 96-98.7) of survey respondents strongly agreed/agreed that the sessions delivered were of high quality.

Discussion The BSPGHAN series has been a positive initiative arising from the pandemic, providing access to high quality PGHAN education when local availability was paused, and giving a platform for the society internationally. Our report shows that the BSPGHAN Education Series has been wellreceived by attendees. The virtual sessions are more accessible compared to in-person teaching sessions, as evidenced by the high percentage of feedback respondents accessing the sessions from home.

Looking ahead, the BSPGHAN Education Group, set up in October 2020, will play a vital role in the further development of the Education Series. Sessions are recorded and made available to BSPGHAN members on the BSPGHAN websitefurther work may include creating online learning modules centred around these recordings. AHP involvement is an area for development- for 2021, we hope to include more topics that will be relevant to their interests.

Acknowledgments We thank all speakers for contributing to the teaching programme, and to all trainees who have devoted their time and efforts towards organising and running the teaching programme.

\section{DUPILUMAB - REPORT OF RESOLUTION OF REFRACTORY EOSINOPHILIC OESOPHAGITIS ALONGSIDE SUCCESSFUL TREATMENT OF ATOPIC ECZEMA}

Kathryn Allan, Aswatha Rabindranathnambi, Jane Ravenscroft, Sabarinathan Loganathan. Nottingham University Hospitals NHS Trust

\subsection{6/flgastro-2021-bspghan.7}

Case We present a male teenager with a background of severe eczema since infancy, multiple food allergies and seasonal allergic rhinoconjunctivitis since early childhood. Systemic immunosuppressants including ciclosporin and methotrexate had failed to control his severe eczema. At the age of 15 years he developed dysphagia associated with difficulty swallowing food. He had no bolus obstruction or vomiting but did experience nausea. He was already on a PPI for suspected gastro-oesophageal reflux. An upper GI endoscopy with biopsies at multiple levels revealed a concentric ring appearance in the mid oesophagus. The histology showed $>30$ eosinophils per high power field with heavy spongiosis; both the macroscopic and microscopic findings being consistent with a diagnosis of eosinophilic oesophagitis (EoE). He was initially treated with oral viscous budesonide but showed no response after 3 months (either clinically or at reassessment endoscopy). He then received an exclusive elemental diet for 10 weeks but still showed no resolution of his EoE.

A multidisciplinary decision with the dermatologists and gastroenterologists was made to stop his methotrexate and elemental diet and to treat with Dupilumab as a single agent, primarily to treat his severe eczema. After 12 months of treatment his eczema had almost completely resolved and his dysphagia was markedly improved. A repeat upper GI endoscopy showed 3-4 eosinophils per high power field, in keeping with adequately treated EoE.

Discussion EoE is a condition strongly associated with food allergies and atopy. Its diagnosis requires the presence of symptoms (including persistent dysphagia, food impaction or GORD that fails to respond to treatment), histological findings of $>15$ eosinophils per high power field in at least 1 biopsy and the exclusion of other causes. The incidence appears to be increasing with males in their 3rd and 4th decade being most commonly affected.

ESPGHAN have designed an algorithm for the recommended treatment for EoE in children and young people. They recommend the use of either topical steroids or an exclusion/elemental diet. If one of these proves ineffective they advise trying the other modality.

EoE is thought to be mediated primarily by food allergies triggering type 2 helper T-cell activity, resulting in release of IL 4, IL 5 and IL 13 cytokines. Dupilumab is a monoclonal antibody which inhibits IL4 and IL13 signalling and has been shown to be effective in control of atopic eczema. A recent randomized controlled trial (RCT) in adults has shown significant improvement in symptoms and endoscopic features of EoE with Dupilumab versus placebo. A phase 3 double-blind RCT evaluating efficacy and safety of Dupilumab vs placebo for EoE in adolescents and adults is ongoing.

Conclusion This is the first paediatric case report of Dupilumab being successfully used to treat both EoE and stubborn eczema which had failed to respond to other immunosuppressants. This was a very complex case due to the extensive atopy since infancy and the need to go beyond the current guidelines to treat his EoE.

\section{IS ANTI-TISSUE TRANSGLUTAMINASE ANTIBODY TITRE GREATER THAN FIVE TIMES UPPER LIMIT OF NORMAL SUITABLE FOR NO-BIOPSY PATHWAY DIAGNOSIS OF COELIAC DISEASE?}

${ }^{1}$ Daniyal Raja, ${ }^{2}$ Dharamveer Basude, ${ }^{3}$ Siba Paul. ${ }^{1}$ University of Exeter - Medical School; ${ }^{2}$ Bristol Royal Hospital for Children; ${ }^{3}$ Yeovil District Hospital

\subsection{6/flgastro-2021-bspghan.8}

Background The coeliac disease (CD) guidelines were updated by ESPGHAN in 2020 confirming that children (0-16yrs) with TGA-IgA titres $\geq 10 \mathrm{x}$ upper limit of normal ( $\geq 10 \mathrm{xULN})$ and positive EMA result can safely be diagnosed with $C D$ via the no-biopsy pathway (NBP). This practice is well adopted in the UK and has led to prompt diagnosis, reduction of the burden on endoscopy services and significant cost saving to the NHS. The COVID-19 pandemic has led to unprecedented challenges for the health service especially endoscopy services. We rarely observed non-diagnostic histopathology TGA-IgA $\geq 5 \mathrm{x}$ ULN in our unit which receives referrals from whole of Southwest England. 


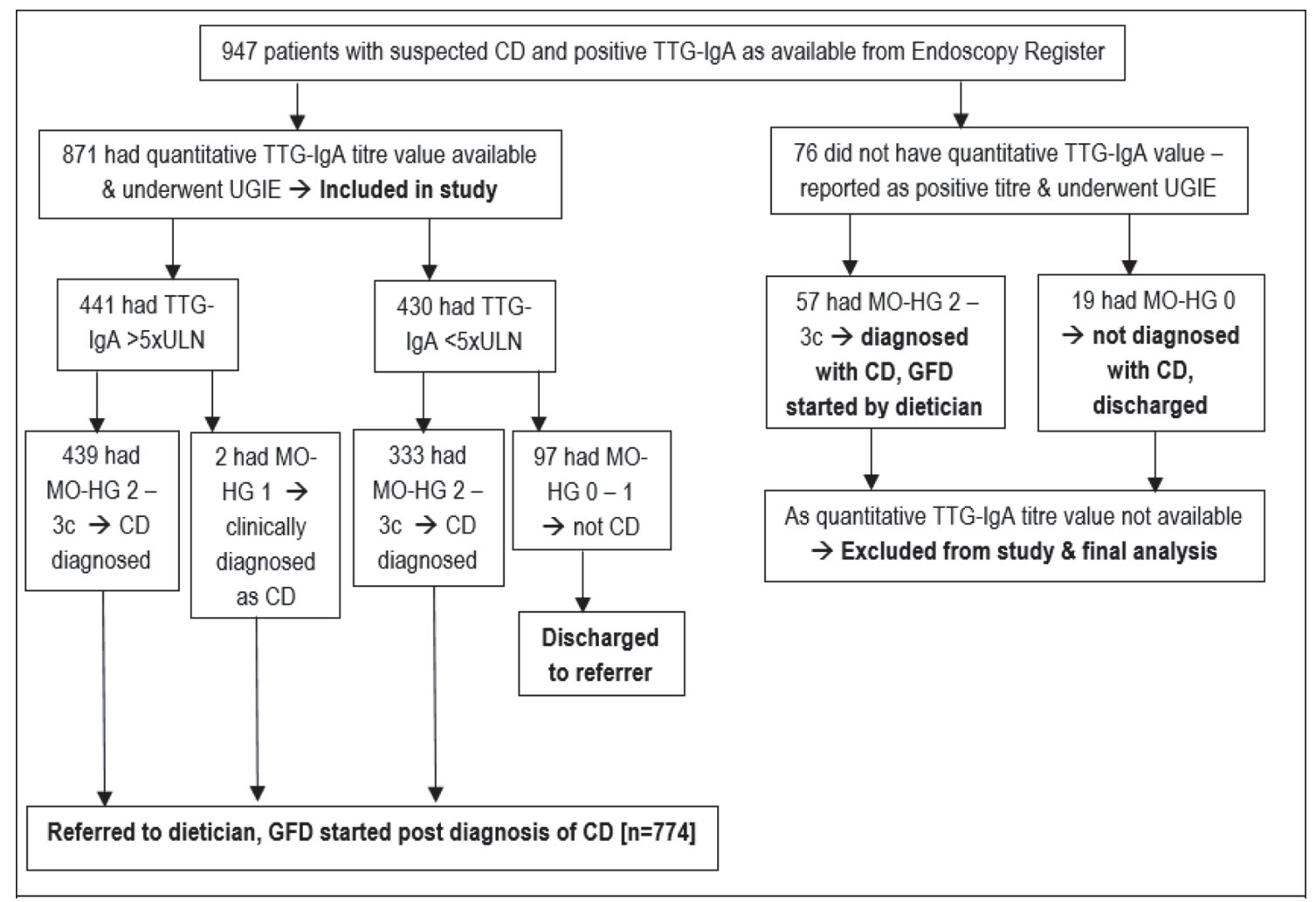

Abstract 08 Figure 1 Flow chart showing TTG-IgA and histological correlation for children with suspected CD.

CD, celiac disease; GFD, gluten free diet; MO-HG, Marsh Oberhuber histological grading; TTG-IgA, IgA-based anti-tissue transglutaminase antibody; UGIE, upper gastrointestinal endoscopy; ULN, upper limit of normal

Aims To explore the relationship of TGA-IgA $\geq 5 \mathrm{x}$ ULN with histological diagnosis of $\mathrm{CD}$ in children referred to a single large tertiary centre.

Methods Prospectively recorded data for children diagnosed with CD following endoscopy over 14-year period (September 2006 to August 2020) was analysed. The data included age, sex, reason for screening, indication for endoscopy, TGA-IgA levels at endoscopy, and histological findings. Where quantitative TGA-IgA was unavailable or not recorded were excluded from the analysis. Statistical analysis was performed using $\chi 2$ analysis and $\mathrm{p}<0.05$ was considered significant.

Results 947 children had endoscopy, but 871 had complete data and were included in final analysis. $772 / 871$ received a histological confirmation of CD by Marsh-Oberhuber histological grading (MO-HG) 2 to $3 \mathrm{c}$. 441 had TGA-IgA $\geq 5 \mathrm{x}$ and $439(99.5 \%)$ had a positive histological diagnosis. The likelihood of a positive biopsy with TGA-IgA $\geq 5 \mathrm{x}$ titre (439/441) compared to TGA-IgA $<5$ ULN titre (333/430) has strong statistical significance $(\mathrm{p}<0.00001)$. Two children of 441 who had MO-HG $<2$ actually had TGA-IgA $>10$ ULN. The mean and median ages of the patients with confirmed CD $(\mathrm{n}=772)$ was 8.68 years and 9.1 years respectively (range $0-17$ years), with a male to female ratio $\approx$ $1: 2$. Figure 1 shows the outcome of the 947 children who had endoscopy.

Conclusion This study showed that $99.5 \%$ of children with TGA-IgA $\geq 5 \times$ ULN had clear histological confirmation of CD with $\mathrm{p}<0.00001$ compared to TGA-IgA $<5 \times \mathrm{xULN}$. For the same advantages of the current NBP and considering the challenges posed by the COVID-19 pandemic, changing the guidance to TGA-IgA $\geq 5 \mathrm{xULN}$ appears to be safe and secure for diagnosis of CD in children.

\section{SUSTAINED CLINICAL AND FINANCIAL BENEFITS OF A NUTRITION SUPPORT TEAM}

Michelle Butcher, Tracey Johnson, Gabis Chana, Sue Protheroe, Theo Wong, Wolfram Haller. Birmingham Women's and Children's hospital

\subsection{6/flgastro-2021-bspghan.9}

Background The evidence-based standard for optimal nutritional support is the multidisciplinary approach of a Nutrition Support Team (NST). Following a pilot study in 2012 showing reduced usage and wastage of parenteral nutrition (PN) a business case was accepted in 2014 to fund the NST at Birmingham Children's Hospital $(\mathrm{BCH})$. The initial focus of the team was to reduce PN usage and between 2012-2016 we reported a reduction in PN days of $20 \%$. There was also an increase in the use of standard bags from $6 \%$ to $14.5 \%$ and a reduction in wastage from $5 \%$ to $3 \%$. Cost savings were estimated to exceed $£ 150,000$. Between $2017-2020$ the NST has continued to work at reducing $\mathrm{PN}$ use but have actively sourced more standard bags and improved education of clinical teams on appropriate use of standard PN.

Aim To assess whether there has been a sustained reduction in overall PN usage, a further increase in standard bag usage and reduction in wastage between 2017-2020.

Methods PN usage and PN wastage data for 2017-2020 was collated using the $\mathrm{BCH}$ pharmacy database. Data included number of PN referrals, total number of PN days per month, $\%$ standard bags and $\%$ wastage. This was compared with data from 2012-2016. Wastage was defined as unused and discarded PN.

Results Mean PN usage has fallen from 752 PN days/month in 2016 to 634 in 2020 showing a further 15\% reduction in 